4. Ganzina F. 4'Epi-Doxorubicin; A new analogue of Doxorubicin: A preliminary overview of preclinical clinical data. Cancer Treat Rep $1983,10,1-22$.

5. Jones WG, Mattsson W. Phase II study of weekly low dose 4 'epidoxorubicin in advanced postmenopausal breast cancer. Cancer Treat Rep 1984, 68, 675-677.

6. Jones WG. Effective palliation of advanced breast cancer with weekly low dose Epirubicin. Eur $\mathcal{J}$ Cancer Clin Oncol 1989, 25, 357-360.

7. Cersosimo RJ, Hong WK. Epirubicin: a review of the Pharmacology, Clinical Activity, and Adverse Effects of an Adriamycin analogue. $\mathcal{F}$ Clin Oncol 1986, 4, 425-439.

8. Sigurdson H, Johansson-Terse I, Aspegren $\mathrm{K}$ et al. Weekly dose doxorubicin (WDA) in advanced breast cancer. Radiother Oncol 1986, 7, 133-139.

9. Mattsson W, Borgstrom S, Landberg T. A weekly schedule of low dose doxorubicin in treatment of advanced breast cancer. Clin Ther $1982,5,193-203$.

10. Tucci E, Algeri R, Guarnieri A, Pepi F, Sapio L, Bastreghi G, Pirtoli L. Weekly Epirubicin in advanced breast cancer. Tumour $1988,74,689-682$.

Eur 7 Cancer, Vol. 26, No. 7, pp. 848-849, 1990

Printed in Great Britain

$0277-5379990 \$ 3.00+0.00$

(c) 1990 Pergamon Press plc

\section{Methylglyoxal bis-Guanylhydrazone in Advanced Bladder Cancer}

\author{
Daniel D. Von Hoff, Brent A. Blumenstein, \\ Theodore W. Pollock, E. David Crawford, \\ James K. Weick, Jerry T. Guy, \\ Mario Eisenberger, William S. Fletcher and \\ Ronald B. Natale
}

Although there has been progress in the development of combination chemotherapy regimens for patients with advanced bladder cancer there is a need for new active agents [1-3]. Methylglyoxal bis-guanylhydrazone (MGBG) is a polyamine biosynthesis inhibitor which induced complete remission in patients with transitional cell carcinoma of the bladder in phase I trials with the agent [4].

46 patients with advanced metastatic transitional cell carcinoma of the bladder were entered into a phase II trial. Eligibility criteria included: histologically confirmed, bidimensionally measurable metastatic transitional cell carcinoma of the bladder; only one previous systemic chemotherapy or immunotherapy regimen (up to two previous intravesical chemotherapy or immunotherapy regimens were acceptable); patients could have had radiotherapy if the disease had progressed (if measurable disease existed outside the previous radiation field); patients had

Correspondence to D.D. Von Hoff, Southwest Oncology Group (SWOG-8519), Operations Office, 5430 Fredericksburg Road, Suite \#618, San Antonio, TX 78229-6197, U.S.A.

D.D. Von Hoff is at the University of Texas Health Science Center at San Antonio, San Antonio, Texas; B.A. Blumenstein is at the Southwest Oncology Group Statistical Center, Seattle, Washington; T.W. Pollock and J.T. Guy are at the Columbus CCOP, Columbus, Ohio; E.D. Crawford is at the University of Colorado, Denver, Colorado; J.K. Weick is at the Cleveland Clinic Foundation, Cleveland, Ohio; $M$. Eisenberger is at the University of Maryland UCOP, Baltimore, Maryland; W.S. Fletcher is at the Oregon Health Sciences University, Portland, Oregon; and R.B. Natale is at the University of Michigan Medical Center, Ann Arbor, Michigan, U.S.A.
Table 1. Patients' characteristics

\begin{tabular}{lr}
\hline Entered & 46 \\
Total eligible (1 patient had no measurable disease) & 45 \\
M/F & $34 / 11$ \\
Median age in years (range) & $61(40-80)$ \\
& \\
Performance status (SWOG) & 11 \\
0 & 16 \\
1 & 13 \\
2 & 5
\end{tabular}

Previous therapy

None

Radiation therapy + chemotherapy

Chemotherapy ${ }^{\star}$ or immunotherapy

1

No. of weeks of therapy

$<4$

4

$5-8$

$>8$

Unknown (too early)

11

16

5

Best response achieved

Complete

Partial

Stable disease

Progression

Assumed no response

No follow-up measurements

Early death

Refused further therapy secondary to toxicity

Too early

*Methotrexate + vinblastine + doxorubicin + cisplatin

to have a SWOG performance status of 3 or less; white cells $3500 / \mu \mathrm{l}$ or more and platelets $100,000 / \mu \mathrm{l}$ or more; serum creatinine $177 \mu \mathrm{mol} / \mathrm{l}$ or less and serum bilirubin $34 \mu \mathrm{mol} / \mathrm{l}$ or less; and patients' informed consent.

MGBG was administered weekly at $600 \mathrm{mg} / \mathrm{m}^{2}$ as an intravenous infusion in $150 \mathrm{ml} \mathrm{D} 5 \mathrm{~W}$ or normal saline over $30 \mathrm{~min}$ or more. Dose escalations of $100 \mathrm{mg} / \mathrm{m}^{2}$ were given if no toxicity was noted. Weekly doses were reduced by $100 \mathrm{mg} / \mathrm{m}^{2}$ for severe (SWOG grade 3) toxicities. One course of therapy was defined as 4 weeks of MGBG. SWOG criteria were used to assess tumour response.

45 of the 46 patients entered were eligible (Table 1). 1 patient had no measurable disease. 20 of the eligible patients (44\%) had at least one or more courses. There were no complete or partial responses. The exact $95 \%$ confidence interval of 0 out of 45 is $0-8 \%$.

Toxicities in the study consisted of grade 3 (severe) or greater nausea and vomiting in $16 \%$ of patients, with 2 patients requiring admission. Grade 3 diarrhoea occurred in $9 \%$ of patients (1 admitted). 1 patient had grade 4 mucositis and 1 had a perforated diverticulum leading to death. Other grade 2 or greater toxicities included fatigue and weakness in 3 patients, hypoglycaemia in 2, hypotension (under $90 \mathrm{mmHg}$ systolic) in 2, weight loss in 4 patients (1 lost $4.5 \mathrm{~kg}$ and 1 lost $7.7 \mathrm{~kg}$ ), and anaemia in 8 . Toxicities were so troublesome that 8 patients refused additional treatments (usually after only 1-3 doses).

Despite the protocol calling for failure on only one previous chemotherapeutic regimen it is clear the patient population was 